\title{
Combining Electrostimulation With Fiber Tracking to Stratify the Inferior Fronto-Occipital Fasciculus
}

\section{OPEN ACCESS}

Edited by: Nico Sollmann, Technical University of Munich, Germany

Reviewed by:

Sandro M. Krieg, Technical University of Munich,

Germany

Michael Sughrue,

University of New South Wales, Australia

*Correspondence: Hugues Duffau hugues.duffau@chu.montpellier.fr

Specialty section: This article was submitted to Brain Imaging Methods, a section of the journal Frontiers in Neuroscience

Received: 20 March 2021 Accepted: 23 April 2021 Published: 20 May 2021

Citation:

Roux A, Lemaitre A-L,

Deverdun J, Ng S, Duffau H and

Herbet G (2021) Combining Electrostimulation With Fiber Tracking to Stratify the Inferior Fronto-Occipital

Fasciculus.

Front. Neurosci. 15:683348. doi: 10.3389/fnins.2021.683348

\begin{abstract}
Alexandre Roux ${ }^{1,2,3}$, Anne-Laure Lemaitre ${ }^{4}$, Jeremy Deverdun ${ }^{5,6}$, Sam Ng $^{4,7}$, Hugues Duffau ${ }^{4,7 *}$ and Guillaume Herbet ${ }^{4,7}$
\end{abstract}

${ }^{1}$ Department of Neurosurgery, GHU Paris, Sainte-Anne Hospital, Paris, France, ${ }^{2}$ Université de Paris, Sorbonne Paris Cité, Paris, France, ${ }^{3}$ Inserm UMR 1266, IMA-Brain, Institute of Psychiatry and Neurosciences of Paris, Paris, France, ${ }^{4}$ Department of Neurosurgery, Gui de Chauliac Hospital, Montpellier University Medical Center, Montpellier, France, ${ }^{5}$ Department of Neuroradiology, Gui de Chauliac Hospital, Montpellier University Medical Center, Montpellier, France, ${ }^{6}$ I2FH, Institut d'Imagerie Fonctionnelle Humaine, Gui de Chauliac Hospital, Montpellier University Medical Center, Montpellier, France, ${ }^{7}$ Institute of Functional Genomics, University of Montpellier, INSERM, CNRS, Montpellier, France

The inferior fronto-occipital fasciculus (IFOF) is one of the longest association fiber tracts of the brain. According to the most recent anatomical studies, it may be formed by several layers, suggesting a role in multiple cognitive functions. However, to date, no attempt has been made to dissociate the functional contribution of the IFOF subpathways. In this study, real-time, cortico-subcortical mapping with direct electrostimulation was performed in 111 patients operated on in wide-awake surgery for a right low-grade glioma. Patients performed two behavioral tasks during stimulation, tapping, respectively, mentalizing and visual semantic cognition-two functions supposed to be partly mediated by the IFOF. Responsive white matter sites were first subjected to a clustering analysis to assess potential topological differences in network organization. Then they were used as seeds to generate streamline tractograms based on the HC1021 diffusion dataset (template-based approach). The tractograms obtained for each function were overlapped and contrasted to determine whether some fiber pathways were more frequently involved in one or the other function. The obtained results not only provided strong evidence for a role of the right IFOF in both functions, but also revealed that the tract is dissociable into two functional strata according to a ventral (semantic) and dorsal (mentalizing) compartmentalization. Besides, they showed a high degree of anatomo-functionnal variability across patients in the functional implication of the IFOF, possibly related to symmetrical/hemispheric differences in network organization. Collectively, these findings support the view that the right IFOF is a functionally multi-layered structure, with nevertheless interindividual variations.

Keywords: diffuse glioma, non-verbal semantic cognition, mentalizing, inferior fronto-occipital fasciculus, electrostimulation

\section{INTRODUCTION}

The inferior fronto-occipital fasciculus (IFOF) is one of the longest association fiber tract of the brain; it interconnects the frontal lobe to different territories of the posterior cortex (Schmahmann and Pandya, 2007). Although the earliest descriptions of the IFOF are longlasting (Burdach, 1822; Dejerine and Dejerine-Klumpke, 1895; Trolard, 1906; Curran, 1909), 
the interest for this ventral fasciculus has renewed only recentlyespecially because of the revival of post-mortem anatomical dissection techniques (Martino et al., 2010; Sarubbo et al., 2013) and the development of tractographic imaging that allows indirect visualization of fiber pathways (Catani et al., 2002; Mori et al., 2002, 2005; Schmahmann and Pandya, 2007; Thiebaut de Schotten et al., 2012; Caverzasi et al., 2014). The IFOF has been thought to be specific to humans, but recent evidence indicates that it may be in fact present in the monkey brain (Sarubbo et al., 2019).

Anatomical studies suggest that the IFOF is not a monolithic white matter (WM) tract, but rather organized into distinct anatomical strata. The first modern dissection work has shown that the IFOF may be split into two layers, a superficial one and a deeper one (Martino et al., 2010). The former connects the frontal lobe to both the superior parietal lobule and the postero-superior part of the occipital lobe, whereas the latter interconnects the frontal lobe with the postero-inferior part of the occipital lobe and the postero-basal temporal areas. More recently, a study combining anatomical dissection and diffusion imaging has allowed disentangling the frontal projections of the two layers (Sarubbo et al., 2013). The superficial layer terminates in the inferior frontal gyrus, while the deep one ends in different prefrontal areas thus forming different subpathways: the anterior sublayer has cortical terminations in both the orbito-frontal cortex and the frontal pole, the middle sublayer in the lateral orbito-frontal cortex and the middle frontal gyrus, and the posterior sublayer mainly in the dorsolateral prefrontal cortex. According to another work using diffusion spectrum imaging, the connective architecture of the IFOF may be even more complex than previously thought, with possibly five layers that would be "trackable" from their prefrontal terminations (Wu et al., 2016). Other studies confirmed that the IFOF projects into many areas of the brain; among them, some were not previously identified as receiving connective inputs from the tract such as the angular gyrus or the posterior temporal cortex (Caverzasi et al., 2014; Hau et al., 2016; Wu et al., 2016). These studies also showed that the IFOF cortical terminations are highly variable across individuals, with the exception of projections targeting the inferior frontal, lateral orbito-frontal, and lateral occipital gyri (Hau et al., 2016).

This multi-layered organization agrees with the array of functions that have been assigned to the IFOF. Neuromodulation studies using direct electrostimulation mapping, the only technique allowing direct access to tract functions (Duffau, 2015), have demonstrated a role of this connection in verbal and visual semantic cognition (Duffau et al., 2005; Moritz-Gasser et al., 2013; Herbet et al., 2017a), and mentalizing (Yordanova et al., 2017), a finding that have been now replicated in lesion mapping works (Almairac et al., 2015; Mirman et al., 2015; Marino Dávolos et al., 2020). Other studies suggested a role in emotion recognition (Philippi et al., 2009; Crespi et al., 2014), spatial cognition and neglect (Urbanski et al., 2008; Herbet et al., 2017b), face recognition (Thomas et al., 2009) and orthographic processes (Vandermosten et al., 2012; Wang et al., 2020). However, neither of these studies has attempted and succeeded to relate the putative functions of the IFOF to specific sublayers.
With the purpose of investigating whether distinct right IFOF sublayers might be specifically devoted to either mentalizing or visual semantic cognition, we analyzed a unique dataset gained from direct brain stimulation mapping achieved in awake patients, combined with q-space diffusion-based fibertrack imaging. The secondary objective was to explore the existence of potential interindividual variations in the functional compartmentalization of the IFOF.

\section{MATERIALS AND METHODS}

\section{Patients}

All patients recruited in this study were consecutively operated on for a histopathologically proven supratentorial diffuse lowgrade glioma at Montpellier University Hospital's Department of Neurosurgery over a period of 5 years. They were included if they met the following criteria: (i) a surgical resection performed in "awake" condition with a cortico-sub-cortical mapping by means of direct electrostimulation; (ii) a glioma close to or infiltrating at least in part the IFOF in the right hemisphere; (iii) an intraoperative mapping of mentalizing and/or visual semantic cognition (see below for a comprehensive description of the behavioral tasks); and (iv) available 3-month postoperative anatomical MRI. Patients showing a preoperative impairment of mentalizing or visual semantic cognition not allowing to objectively probe these functions during the mapping were excluded (no patients were excluded by this criterion).

A total of 189 patients with a right supratentorial low-grade diffuse glioma were initially screened. Among those, we excluded 71 patients for whom the IFOF was not stimulated during the surgical procedure because the tumors were distant from the tract and its interconnected areas. The final sample thus consisted of 111 patients (46 females and 65 males), with a mean \pm SD age of $39.8 \pm 10.4$ years (range: $23-70$ years). All patients except 18 were right-handed (5 ambidextrous and 13 left-handed).

\section{"Awake" Surgery Procedure}

All patients included in this study benefited from a "wide-awake" surgical procedure performed by the same well-experienced neurosurgeon (HD). An intraoperative multifunctional mapping was systematically achieved at both the cortical and axonal levels by means of direct electrostimulation. The general principles of such a surgical approach has been comprehensively described by our research group in another works (Duffau et al., 2005; Tate et al., 2014). Briefly, after craniotomy and dural opening under general anaesthesia, the patient was awoken and the cortical surface was exposed. Next, the tumor gross limits were identified by intraoperative ultrasound and visually indicated with sterile letter tags, placed directly on the cortex. Direct electrical stimulations were then performed using a biphasic electric current $(60 \mathrm{~Hz}$ frequency, $1 \mathrm{~ms}$ pulse-width, amplitude 2-5 $\mathrm{mA}$ ) delivered by a bipolar probe with $5 \mathrm{~mm}$ inter-tip space (NIMBUS Stimulator, Newmedic, France). A sensorimotor and speech mapping was first performed. A small current was initially used and incrementally increased up by step of 0.5 $\mathrm{mA}$ until a reliable speech arrest, or motor or sensory response 
was triggered. This stimulation threshold was maintained during the remaining of the cortical and subcortical mapping. The cerebral cortex was then mapped with different behavioral tasks, including tasks tapping into aspects of mentalizing and visual semantic cognition. To both limit the spread of current through the brain tissue and preserve response specificity, the length of stimulation never exceeded 4 s. All the exposed cortical surface was mapped, and cortical areas whose stimulations induced three non-consecutive, identical perturbations was accepted as responsive for the function under scrutiny (Ojemann and Mateer, 1979); they were subsequently marked with sterile tags. Patients' functions were monitored by a senior clinical neuropsychologist, blinded to when stimulations were applied. After the completion of cortical mapping, several photographs were taken for off-line treatment of data. Then, glioma removal was started. With the progress of resection, the WM tracts were gradually accessible for stimulation. WM sites associated with recurrent functional responses were marked with sterile tags. New photographs from different angles of view were taken.

\section{Intraoperative Behavioral Paradigms}

The behavioral paradigm to assess mentalizing "on-line" has been previously described in another study (Herbet et al., 2015) and is schematically illustrated in Figure 1A. It is an adapted version of the well-used "Read the Mind in the Eyes" task (hereafter, RME), initially developed by Baron-Cohen et al. (2001) to gauge social intelligence in autistic patients. In the standard version of the task, the participant is asked to choose among four complex mental states the one that best matches with what the person seen on a photograph is feeling or thinking. Only the eye region of the face is shown. This version, composed of 36 items, was systematically achieved the day before surgery. Due to the inherent complexity of the RME task, only two mental states were used during the intraoperative mapping in order to decrease the number of false positive manifestations and thus the number of required stimulations. For the same reason, only succeeded items during the preoperative assessment were included in the intraoperative protocol.

To assess visual semantic cognition, the Pyramids and Palm Trees test (hereafter, PPTT) was used (Howard et al., 1992). This force-choice task, in which the participant is required to form a semantic association, comprises 52 black and white items in its standard version. For each target item, two further pictures are proposed. The patient is asked to select the one that best matches with the target picture according to the most straightforward semantic link. As for the RME task, the PPTT was achieved the day before surgery, and impaired patients were dismissed from the mapping. Items associated with incorrect responses were excluded from the intraoperative materials. The intraoperative protocol is described in Figure 1B.

To demonstrate specificity, and depending on which networks were damaged by the tumor, anatomical sites associated with mentalizing and/or visual semantic impairments were also stimulated while the patients were performing other behavior tasks. This included a naming task (Duffau et al., 2005), a line bisection task (Herbet et al., 2017b), a motor task (Rech et al.,
2019), and a passive somatosensory task (Tate et al., 2014). In this study, only semantic and mentalizing sites are reported.

It is worth mentioning that, on the total of patients included $(n=111), 104(93.7 \%)$ patients were intraoperatively assessed for visual semantic cognition, 90 (81.1\%) for mentalizing and 83 (74.8\%) for both functions.

\section{Imaging Acquisition and Spatial Normalization}

Structural MRI datasets were collected 3 months after surgery on either a 1.5T (Avanto, Siemens Medical Systems, Erlangen, Germany) or a 3T MR imaging scanner (Skyra, Siemens Medical Systems, Erlangen, Germany), as part of the routine management protocol. For the purpose of the current work, standard 3D gadolinium-enhanced axial T1-weighted images were used. The $1.5 \mathrm{~T} / 3 \mathrm{~T}$ MRI parameters were as follows: repetition time $1,880 / 1,700 \mathrm{~ms}$; echo time $3.4 / 2.5 \mathrm{~ms}$; inversion time $1,100 / 922 \mathrm{~ms}$; field of view $256 \times 256 / 250 \times 250 \mathrm{~mm}$; flip angle $15 / 9^{\circ}$; voxel size $1 \times 1 \times 1 \mathrm{~mm}^{3}$ and; 176 axial slices.

All individual MRI datasets were registered to the Montreal Neurological Institute (MNI) 152 template with an isotropic resolution of $1-\mathrm{mm}$. This work was achieved using SPM12 software $^{1}$ implemented in MATLAB environment (Matrix Laboratory, MathWorks, Inc.-R2018a), with standard parameters. At this stage, all MRIs were visually inspected to ensure sufficient quality. All registrations were satisfactory.

\section{Distribution of Stimulation-Related Anatomical Sites}

The coordinates of each anatomical site was manually plotted onto the patients' respective normalized MRI. They were established by using a combination of intraoperative photos taken from different points of view (allowing to precisely identify the location of functional sites indicated by sterile numbered tags), written surgical reports, cortical (e.g., gyri, sulci and vessels) and subcortical (e.g., edges of the resection cavity) landmarks identifiable on both the normalized MRI and the intraoperative photos. This method has been successfully applied in recent studies (e.g., Sarubbo et al., 2020). The accuracy of coordinate positioning was systematically checked by the senior author. When a disagreement occurred, the location of problematic sites was remapped in a consensual manner. The MNI coordinates $(x, y, z)$ were recorded in separate databases as a function of the behavior tasks (i.e., PPTT or RME or both) and the stimulation location (i.e., cortical vs. subcortical). Then, for each stimulation site, a spherical volume of interest (VOI) was generated using the MarsBaR toolbox ${ }^{2}$ with a radius of $2.5 \mathrm{~mm}$ corresponding to the spatial resolution of the bipolar stimulator.

To demonstrate reliability in the way stimulation sites were positioned, we asked an independent operator with certified expertise in neurosurgery and neuroanatomy, and fully blind to both the general purpose and the results of the study, to position again 40 subcortical sites randomly selected among the

\footnotetext{
${ }^{1}$ http://www.fil.ion.ucl.ac.uk/spm/

${ }^{2}$ http://marsbar.sourceforge.net
} 
A Reading the Mind in the Eyes Test

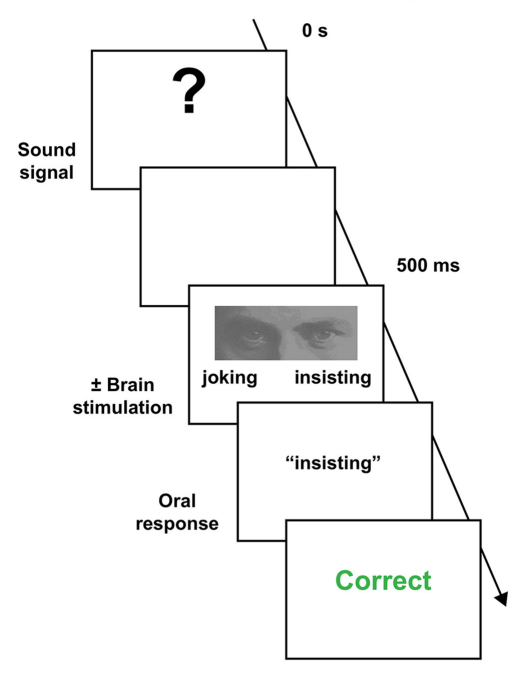

B Pyramids and Palm Trees Test

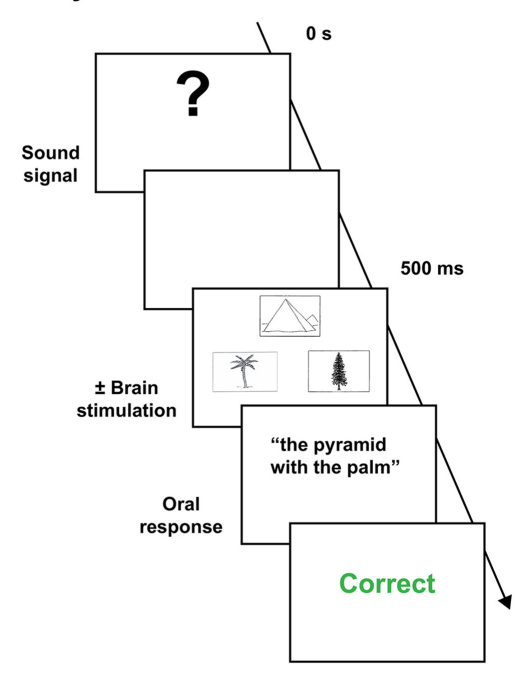

FIGURE 1 | Behavior paradigms employed during the intraoperative mapping. (A) Mentalizing task. (B) Visual semantic cognition task (see the main text to a complete description of the behavioral procedure).

105 subcortical sites already positioned by the first operator (i.e., subcortical sites either associated with mentalizing or visual semantic cognition during electrostimulation). To obtain a quantitative index of inter-operator reliability, three parametric correlations were performed, one for each axis. We further statistically compared the coordinates between both operators. We also calculated both the mean and the standard deviation of the absolute difference in site estimates (one for each axis) in order to provide a measure of error (see Herbet et al., 2019 for a comparable method).

\section{Clustering Analysis of Subcortical Data}

In view of the apparent topological heterogeneity between the distribution of the mentalizing and the semantic subcortical sites, at least in the WM deep in the prefrontal and the posterior parietal cortices (see below), a clustering analysis was conducted with each stimulation dataset. As a first step, we used the DudaHart procedure (Duda and Hart, 1973) to decide whether the data should be split into two or more clusters. Then, when the null hypothesis of homogeneity was rejected at $p<0.05$, we performed a formal clustering analysis with the $k$-means algorithm, by varying $k$ from 2 to 10 with 100 iterations. Clusters were formed by observations that maximize between-cluster distances using a squared Euclidean distance-based measure. The optimal number of clusters was estimated with a leaveone-out cross-validation procedure. To assess the statistical differences between the clusters in each stimulation dataset, an ANOVA was performed for each coordinate. The analyses were mainly achieved with SPSS ${ }^{3}$ and the "Flexible Procedures for

${ }^{3}$ https://www.ibm.com
Clustering” (FPC) package (Hennig, 2020) implemented in $R$ environment ${ }^{4}$.

\section{Disconnection Analysis: A Population-Based Approach With the HCP-1021 Diffusion Data}

Our aim here was to dissociate the WM fiber connections specific either to mentalizing or visual semantic cognition. As a first step, a population-based template was constructed based on the diffusion data of 1,021 participants from the Human Connectome Project (2017 Q4, 1,200-subject release). A multishell diffusion scheme was used; the $b$-values were $1,000,2,000$, and 3,000 $\mathrm{s} / \mathrm{mm}^{2}$. The number of diffusion-sampling directions was 90 for each $b$-value. The in-plane resolution, as well as the slice thickness, was $1.25 \mathrm{~mm}$. The diffusion data were reconstructed in the MNI space using $q$-space diffeomorphic reconstruction (Yeh and Tseng, 2011) to obtain the spin distribution function (Yeh et al., 2010). A diffusion sampling length ratio of 2.5 was used, and the output resolution was $2 \mathrm{~mm}$. The restricted diffusion was quantified using restricted diffusion imaging (Yeh et al., 2017). The analysis was conducted using DSI Studio ${ }^{5}$.

Each 5-mm (in diameter) subcortical spherical VOI was used as seeds for fiber tracking allowing us to generate a map of the fiber connections passing through or originating from this VOI. The same tracking parameters were maintained across all tractographies performed; this included a tracking threshold automatically determined using the Otsu's method, an angular threshold of $55^{\circ}$, a step size of $1 \mathrm{~mm}$, a minimal and maximal length of 10 and $300 \mathrm{~mm}$, respectively. To estimate

\footnotetext{
${ }^{4}$ https://R-project.org/

${ }^{5}$ http://dsi-studio.labsolver.org
} 
fiber direction, the standard trilinear interpolation method was selected. Streamlines were tracked using the Euler Algorithm.

Once all tractographies were performed, they were transformed into binarized maps. These maps were overlaid according to the associated impairment (i.e., mentalizing or visual semantic cognition) using MRIcron software. Then MRIcron's subtraction plot function was employed to identify the WM voxels the most frequently and specifically "disconnected" during mentalizing vs. semantic electrostimulation, and vice versa.

\section{Standard Protocol Approvals, Registrations and Patient Consents}

Data were acquired as part of the current clinical practice and were retrospectively studied. Patients consented to the extraction of data from their medical records. We conducted this study in compliance with the ethical standards of our institution for a retrospective study.

\section{RESULTS}

\section{Patients' Clinical Characteristics and Tumor Topography}

A total of 111 patients (46 females) presenting with a right supratentorial diffuse low-grade glioma fulfilled all inclusion criteria and thus constituted the final sample. An overview of the patients' sociodemographic and clinical data is provided in Table 1. Consistent with the preferential distribution of lowgrade glioma (Herbet et al., 2016), the most common location was in the frontal and the insular cortex. The mean pre-operative tumor volume was $51.0 \pm 41.7 \mathrm{~cm}^{3}$.

\section{Inter-Operator Reliability in Site Estimates}

The results from correlation analyses performed between the MNI coordinates of each operator indicated $r_{40}=0.93, r_{40}=0.99$ and $r_{40}=0.97$ for, respectively, the $x$-, $y$-, and $z$-axis. No significant difference was found in estimates between both operators for the $x$ - $\left(t_{39}=0.14, p=0.89\right), y-\left(t_{39}=1.57, p=0.12\right)$ and $z$-axis $\left(t_{39}=0.90, p=0.37\right)$. The mean $\pm \mathrm{SD}$ of the absolute difference between both operators was $0.08 \mathrm{~mm} \pm 3.36$ for the $x$-axis, $1.27 \mathrm{~mm} \pm 5.12$ for the $y$-axis, and $0.87 \mathrm{~mm} \pm 6.1$ for the $z$-axis. These analyses clearly indicate that the positioning work was reliable and accurate.

\section{Topological Organization of Cortical Sites}

In total, 101 cortical sites were identified across patients; among these, 57 were specifically associated with mentalizing (Figure 2, orange circles), 41 with visual semantic cognition (Figure 2, blue circles) and 3 with both functions (called "aspecific sites") (Figure 2, red circles). Mentalizing sites were distributed in IFG (pars opercularis, $n=24$; pars triangularis, $n=10$; pars orbitalis, $n=1)$, in dlPFC $(n=20)$ and in the superior temporal gyrus (STG, $n=2)$. Semantic sites were mainly located in dlPFC $(n=30)$, and to a lesser extent in the mid-to-posterior STG $(n=7)$ and in IFG
TABLE 1 | Main characteristics of the study sample.

\begin{tabular}{|c|c|c|}
\hline Parameters & $n$ & $\%$ \\
\hline \multicolumn{3}{|c|}{ Clinical characteristics at diagnosis } \\
\hline \multicolumn{3}{|l|}{ Sex } \\
\hline Female & 46 & 41.4 \\
\hline Male & 65 & 58.6 \\
\hline \multicolumn{3}{|c|}{ Age, years (mean, SD) $39.8 \pm 10.4$} \\
\hline$\leq 40$ & 64 & 57.7 \\
\hline$>40$ & 47 & 42.3 \\
\hline \multicolumn{3}{|l|}{ Handedness } \\
\hline Right-handed & 93 & 83.8 \\
\hline Left-handed & 13 & 11.7 \\
\hline Ambidextrous & 5 & 4.5 \\
\hline \multicolumn{3}{|l|}{ Presenting symptom } \\
\hline Incidental & 29 & 26.1 \\
\hline Epileptic seizure & 80 & 72.1 \\
\hline Neurological focal deficit & 2 & 1.8 \\
\hline \multicolumn{3}{|c|}{ Imaging characteristics at diagnosis } \\
\hline \multicolumn{3}{|l|}{ Topography of tumor } \\
\hline Frontal & 45 & 40.6 \\
\hline Fronto-temporo-insular & 25 & 22.5 \\
\hline Temporal & 12 & 10.8 \\
\hline Parietal & 7 & 6.3 \\
\hline Fronto-insular & 6 & 5.4 \\
\hline Temporo-insular & 4 & 3.6 \\
\hline Temporo-occipital & 3 & 2.7 \\
\hline Insular & 3 & 2.7 \\
\hline Fronto-cingular & 3 & 2.7 \\
\hline Fronto-temporo-parieto-insular & 2 & 1.8 \\
\hline Fronto-parieto-insular & 1 & 0.9 \\
\hline \multicolumn{3}{|c|}{ Tumor volume, $\mathrm{cm}^{3}$ (mean, SD) $51.0 \pm 41.7$} \\
\hline$<50$ & 67 & 60.4 \\
\hline$\geq 50$ & 44 & 39.6 \\
\hline \multicolumn{3}{|c|}{ Treatment-related characteristics } \\
\hline \multicolumn{3}{|c|}{ Extent of resection, percent (mean, SD) $92.8 \pm 8.8$} \\
\hline$<90 \%$ & 29 & 26.1 \\
\hline$\geq 90 \%$ & 82 & 73.9 \\
\hline
\end{tabular}

(pars opercularis, $n=1$; pars triangularis, $n=3$ ). Among the three "aspecific" sites, two were located in STG and one in dIPFC. To summarize, the spatial distribution of mentalizing and semantic sites were relatively similar across patients, except for IFG that was clearly under-represented for visual semantic cognition.

\section{Topological Organization of Subcortical Sites}

Overall, 105 sites were detected during subcortical stimulation: 46 were specific to mentalizing, 49 to semantic cognition, and 10 were associated with both functions. The distribution of these anatomical sites is provided in Figure $\mathbf{3 A}$, the vast majority of which were located along the topological positioning of the IFOF according to the most recent anatomical studies using either DTI (Figure 3B) or post-mortem dissection (Figure 3C). Visually, although the two distributions were quite similar, though, two differences were noticeable: indeed, mentalizing sites 


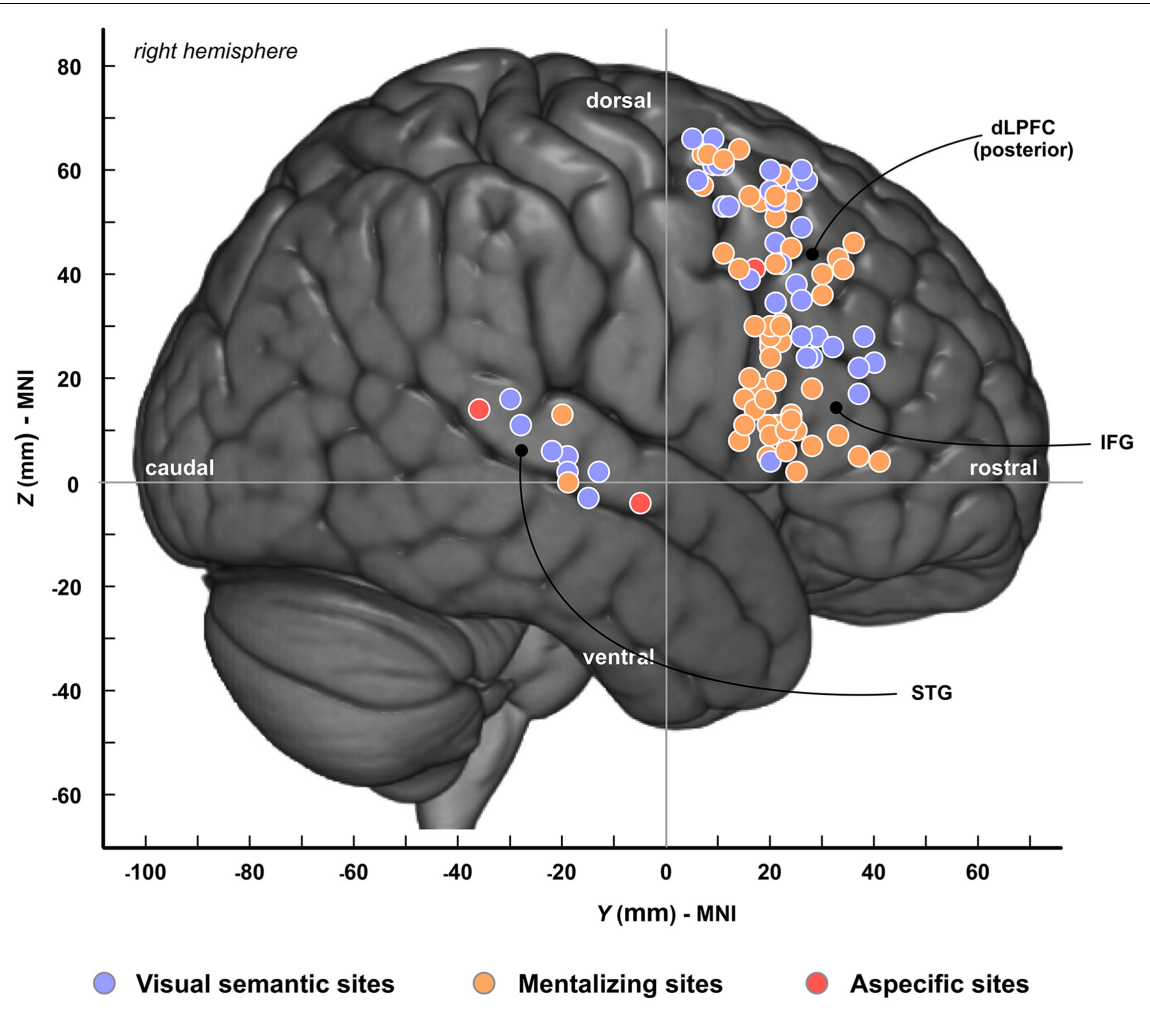

FIGURE 2 | Spatial topography of cortical sites. Cortical sites are plotted onto the MNI-152 template. Colors refer to the type of neuropsychological impairments induced by electrostimulation. Blue, visual semantic cognition; Orange, mentalizing; Red, sites associated with both visual semantic cognition and mentalizing. dIPFC, dorsolateral prefrontal cortex; IFG, inferior frontal gyrus; STG, superior temporal gyrus.

were over-represented in the WM fibers underlying the anterior part of both dlPFC and pars triangularis whereas visual semantic cognition were rather over-represented in the WM fibers of the posterior parietal cortex (Figure 3A).

\section{Relationships Between Cortical and Subcortical Sites, and Between Functions}

In total, 83 patients benefited from a mapping concerning both mentalizing and visual semantic cognition. At the cortical level, at least one anatomical site for each function was identified in 16 patients (19.3\%). Conversely, 14 (16.9\%) patients uniquely showed one (or more) site(s) for visual semantic cognition, and 35 (42.2\%) for mentalizing. Note that no cortical responses were found in 18 patients (21.6\%) for these functions.

As regards with subcortical mapping, 16 patients (19.3\%) showed at least one anatomical site for each function. 16 (19.3\%) patients uniquely showed one (or more) site(s) for visual semantic cognition, and 33 (39.8\%) for mentalizing. No subcortical responses were found in 18 patients $(21.6 \%)$.

Collectively, these results show a high inter-individual variability in the functional involvement of the IFOF with, nevertheless, a more frequent implication for mentalizing vs. visual semantics (2-tailed $t$-test for proportion comparison, $p=0.004)$. Interestingly, this variability echoed those identified at the cortical level with, again, an over-involvement of mentalizing vs. visual semantic cognition $(p=0.002)$.

As a significant proportion of patients was left-handed, we checked whether the distribution of functional sites could be considered as equivalent between left-handed and right-handed patients. The proportion of unmasked cortical sites were not different for mentalizing $(p=0.46)$ and visual semantic cognition ( $p=0.78)$. However, although no difference was revealed for semantic cognition at the subcortical level $(p=0.21)$, this was not the case for mentalizing $(p=0.017)$ (left-handed patients showed a lower rate of subcortical sites). This suggests that the right white matter network is less engaged in left-handed patients for mentalizing.

\section{Clustering Analysis Results}

Both mentalizing and non-verbal semantic cognition stimulation datasets were allowed to be clustered according the Duda-Hart statistics $(p<0.05)$. For mentalizing, a 5 -cluster solution model was found to best fit the data (training error $=0.037$ ). The MNI coordinates between the five clusters were significantly different $(x: F=559.01, p<0.001 ; y: F=77.48, p<0.001$; $z: F=104.16, p<0.001)$. For visual semantic cognition, a 6cluster solution model was estimated as the most appropriate for the data (training error $=0.033$ ) and, again, the betweencoordinates differences were significant $(x: F=326.28, p<0.001$; $y: F=47.18, p<0.001 ; z: F=99.68, p<0.001)$. The 

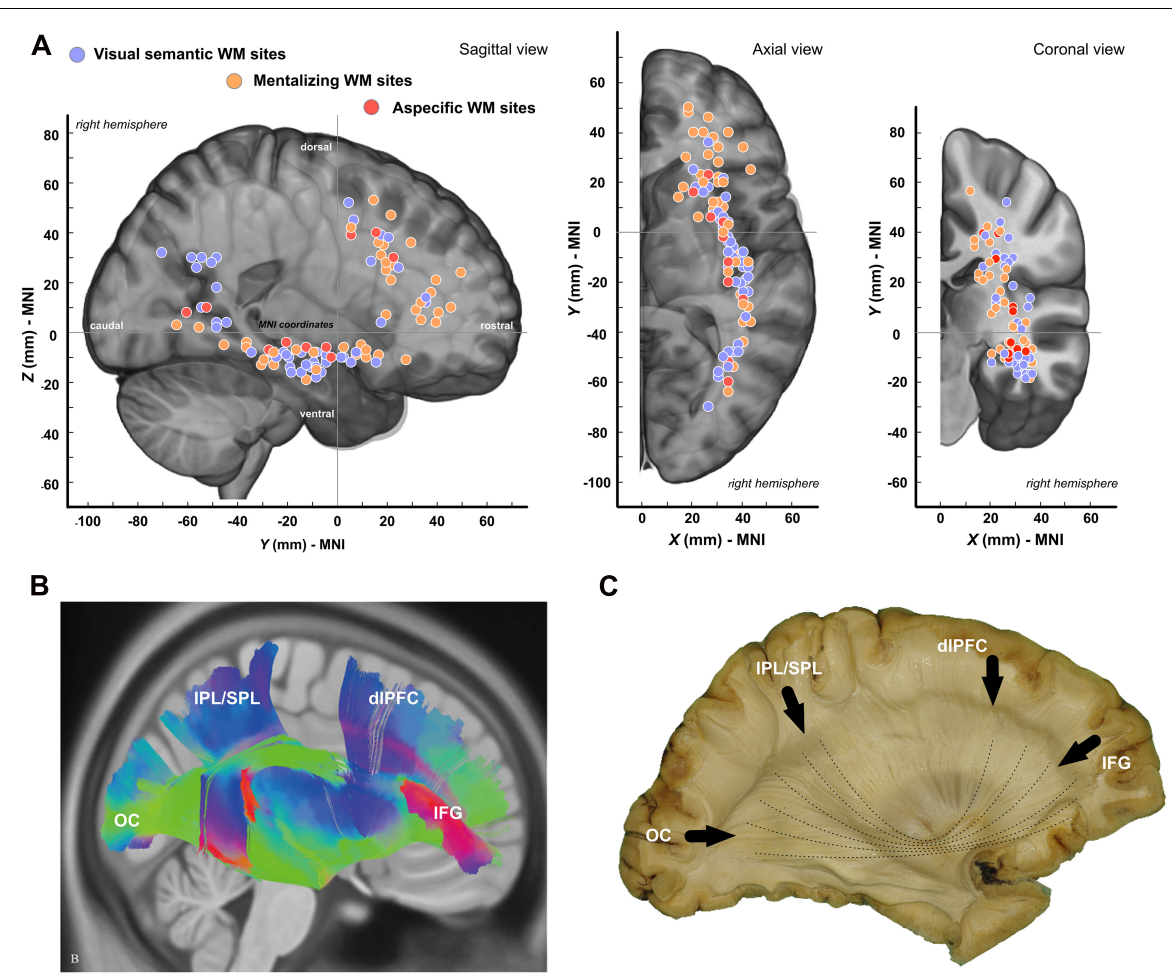

FIGURE 3 | Spatial distribution of white matter sites. (A) Tridimensional distribution of WM sites. (B) Template-based fiber tracking of the right IFOF with q-diffusion imaging (from Wu et al., 2016). (C) Anatomical dissection of the right IFOF (personal materials). OC, occipital pole; IPL, inferior parietal lobule; SPL, superior parietal lobule; dIPFC, dorsolateral prefrontal Cortex; IFG, inferior frontal gyrus.

centroids \pm SD of the clusters are reported in Table 2 and plotted in Figure 4. To summarize, a high topological similarity was found for both functions in the prefrontal dorsolateral, subinsular/anterior extreme capsule and temporal WM. Dissimilarities were, however, observed in the WM of the antero-dorsal pars triangularis (cluster specific to mentalizing) and in WM of the angular gyrus (cluster specific to visual semantic cognition).

\section{Disconnection Analysis}

The disconnection analysis, summarized in Figure 5, first confirmed that the most "disconnected" voxels during stimulations associated with either mentalizing or visual semantic cognition mainly belonged to the right IFOF. However, the subtraction between mentalizing and visual semantic cognition binarized streamline tractograms (and vice versa) revealed some noticeable differences: WM semantic sites were more frequently crossed by orbitofrontal and mid-to-post temporal IFOF streamlines whereas WM mentalizing sites were more frequently crossed by fronto-parietal and dorsal temporal stem/extreme/external capsule streamlines. These differences were moderate because not exceeding $25 \%$.

\section{DISCUSSION}

By virtue of their brain-wide cortical projections, association fiber pathways are vital building blocks in integrating information within and across neurocognitive networks (Herbet and Duffau, 2020). Until recently, association tracts were frequently viewed as monolithic structures, the primary consequence of which was a tendency to conceptualize them as monofunctional pieces. However, recent works using tractography imaging or anatomical dissection has provided evidence that association tracts are in fact formed by multiple anatomical layers and thus are possibly involved in different functions (Martino et al., 2010; Wu et al., 2016; Latini et al., 2017; Herbet et al., 2018). The IFOF is no exception to the rule, as its connective architecture is complex. This tract may indeed be segregated into two (Martino et al., 2010; Sarubbo et al., 2013) or more (Wu et al., 2016) sublayers with distinct frontal or posterior terminations. In this context, the present study was an attempt to dissociate two functional pathways into the right IFOF with the major advantage of using stimulation mapping - the sole available method allowing direct functional interrogation of WM tracts (Duffau, 2015).

First of all, the results confirmed that the right IFOF is a critical WM connection in the networks supporting, respectively, visual semantic cognition and mentalizing, as suggested in other neuromodulation (Moritz-Gasser et al., 2013; Herbet et al., 2017a; Yordanova et al., 2017) or neuroimaging studies (Mirman et al., 2015; Grosse Wiesmann et al., 2017). Cortical sites were indeed observed in structures known to receive connective inputs from the IFOF, including posterior dlPFC, IFG and STG (Sarubbo et al., 2013; Caverzasi et al., 2014; Hau et al., 2016; Wu et al., 2016). Most importantly, the subcortical sites were mainly situated 
TABLE 2 | Cluster coordinates.

\begin{tabular}{|c|c|c|c|c|c|c|}
\hline \multirow[t]{2}{*}{ Parameters } & \multirow[t]{2}{*}{ Clusters } & \multirow[t]{2}{*}{ Clusters location } & \multirow[t]{2}{*}{ Number of cases for clustering } & \multicolumn{3}{|c|}{ MNI coordinates \pm SD } \\
\hline & & & & $x$ & $Y$ & $z$ \\
\hline \multirow[t]{5}{*}{ Mentalizing } & Cluster 1 & Temporal & 8 & $35 \pm 3$ & $-9 \pm 7$ & $-11 \pm 4$ \\
\hline & Cluster 2 & Antero-dorsal pars triangularis & 9 & $24 \pm 5$ & $37 \pm 11$ & $16 \pm 6$ \\
\hline & Cluster 3 & Posterior dIPFC & 11 & $23 \pm 6$ & $18 \pm 7$ & $36 \pm 9$ \\
\hline & Cluster 4 & Subinsular/anterior extreme capsule & 8 & $31 \pm 5$ & $20 \pm 12$ & $-5 \pm 6$ \\
\hline & Cluster 5 & Sagittal stratum & 10 & $40 \pm 3$ & $-37 \pm 13$ & $-6 \pm 5$ \\
\hline \multirow[t]{6}{*}{ Visual semantic cognition } & Cluster 1 & Posterior dIPFC & 6 & $28 \pm 6$ & $15 \pm 8$ & $38 \pm 10$ \\
\hline & Cluster 2 & Subinsular/anterior extreme capsule & 4 & $26 \pm 1$ & $27 \pm 11$ & $5 \pm 12$ \\
\hline & Cluster 3 & Temporal & 13 & $41 \pm 2$ & $-19 \pm 7$ & $-12 \pm 4$ \\
\hline & Cluster 4 & Sagittal stratum & 5 & $36 \pm 2$ & $-49 \pm 4$ & $8 \pm 7$ \\
\hline & Cluster 5 & Temporal & 15 & $34 \pm 2$ & $-3 \pm 6$ & $-11 \pm 2$ \\
\hline & Cluster 6 & Angular gyrus & 6 & $31 \pm 3$ & $-56 \pm 8$ & $29 \pm 2$ \\
\hline
\end{tabular}

SD, standard deviation; dIPFC, Dorsolateral Prefrontal Cortex; MNI, Montréal Neurological Institute.
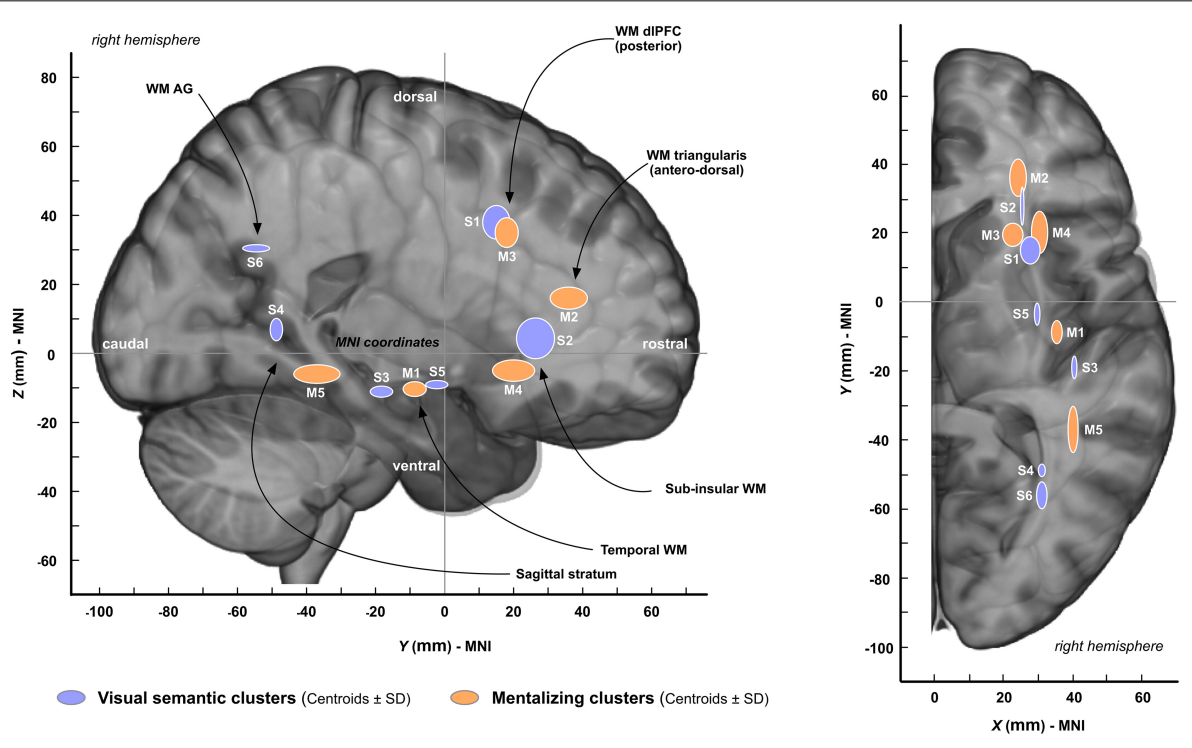

FIGURE 4 | Results from the clustering analysis. The coordinates of each cluster are represented by centroids \pm SD (see Table 2 for the precise MNI coordinates). "S" means "semantic cluster" and "M" means "mentalizing cluster." AG, angular gyrus; dIPFC, dorsolateral prefrontal cortex.

along the typical course of the IFOF (Figure 3). Furthermore, the overlap maps of individual tractograms showed that streamlines generated from subcortical sites belonged in average to the right IFOF, again, for both functions. As matter of fact, the maximum overlaps occurred at the level of the temporal stem, a classical anatomical reference not only to identify the tract during neurosurgery (Duffau et al., 2005) but also to track it with diffusion imaging (steam-based approach) - as the entire range of IFOF fibers pass through this region (Hau et al., 2016; Wu et al., 2016).

The main goal of this study was to determine whether the IFOF might be dissociated into (at least) two anatomical strata, each of them specifically devoted either to semantic cognition or to mentalizing. The results obtained support at least partially this hypothesis. First, consistent with the general distribution of subcortical sites (Figure 3), subcortical clustering analyses revealed that mentalizing sites were over-represented deep in the white matter underlying pars triangularis whereas semantic sites were rather over-represented in the depth of the angular gyrus, suggesting that the IFOF fibers involved in each function are dissociable, but only at the level of the terminal parts of the tract (Figure 4). Second, the subtraction analyses indicated (i) that semantic sites were more frequently crossed by ventral streamlines compared to mentalizing sites, including IFOF orbito-frontal and mid-to-posterior temporal fibers; (ii) conversely that mentalizing sites were more frequently crossed by more dorsal streamlines compared to semantic sites, including fibers passing through the dorsal part of the temporal stem, fibers in the depth of pars triangularis, and fibers belonging to the superior longitudinal fasciculus (SLF) (Figure 5). This pattern of result suggests a functional compartmentalization of the IFOF, with distinct sublayers at the subcortical level (stem 


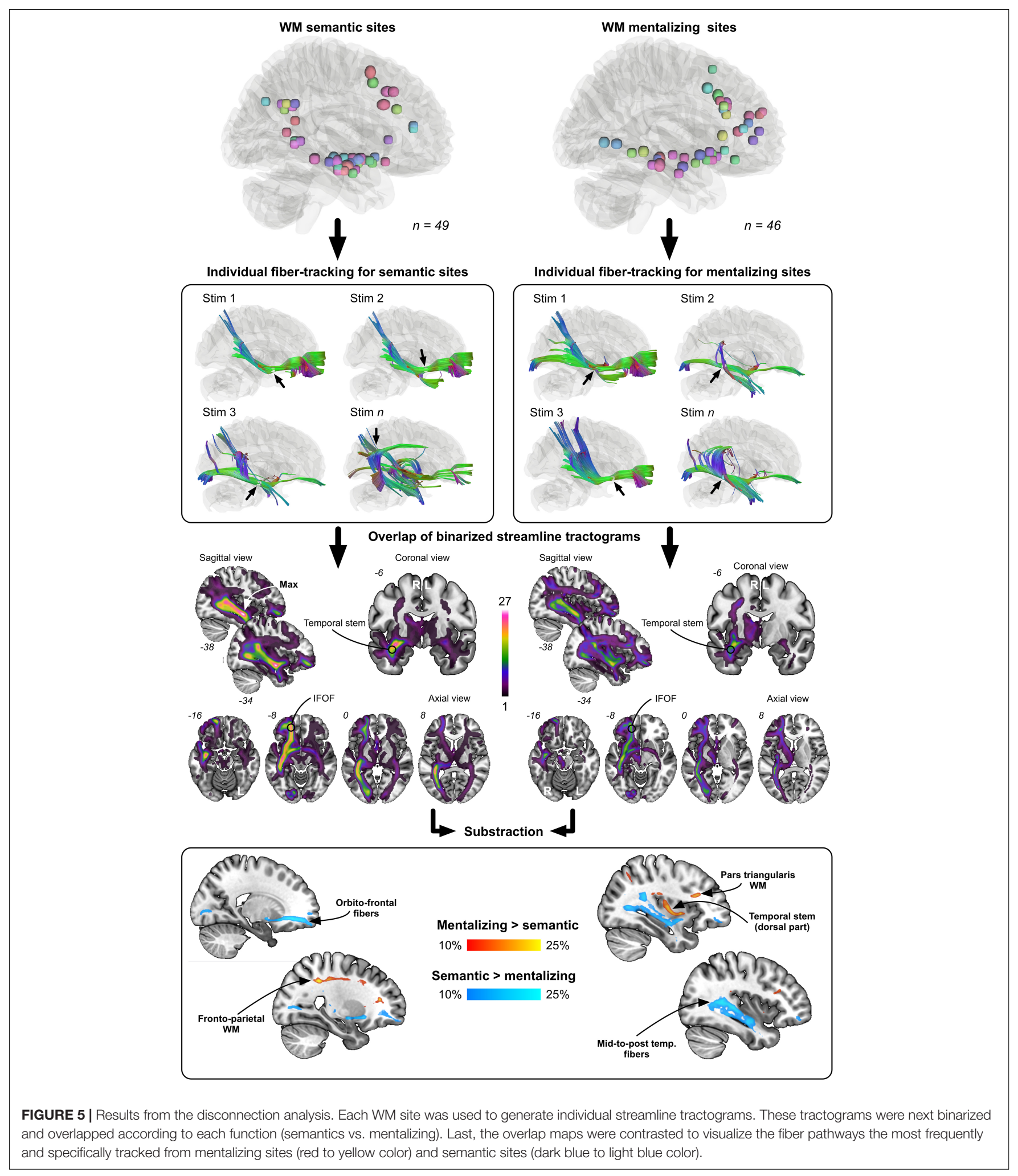

of the bundle) as well as regarding their terminal parts. More specifically, a "ventral" IFOF sublayer may be implicated in visual semantic processing whereas a "dorsal" IFOF layer may be rather implicated in mentalizing with an additional contribution of the
SLF, as previously suggested in lesion mapping studies (Herbet et al., 2014; Grosse Wiesmann et al., 2017).

This interpretation may be criticized on the ground that the subtraction analyses revealed only low to moderate differences 
between streamline distributions associated with semantic cognition vs. mentalizing and vice versa ( $\max 25 \%$ ). This was, however, expected for anatomical reasons. Indeed, a large proportion of stimulation sites was unmasked within the temporal stem. Given the width of this bottleneck structure [around $11 \mathrm{~mm}$ at the level of the limen insulae (Martino et al., 2010)] and the spatial resolution of the bipolar stimulator (5 $\mathrm{mm}$ ), dissociating IFOF WM subpathways in this area is actually challenging. Besides, as reported by $\mathrm{Wu}$ et al. (2016), the fibers forming the different IFOF layers are relatively intermingled in the temporal stem, not organized into wellstructured bundles. Consequently, the difference we found in the subtraction analyses were not so large because related to subcortical sites (and their associated streamlines) located outside the temporal stem, which were clearly less numerous. Note that in a recent study aimed at disentangling the motor-speech from the lexical semantic fiber pathways, comparable differences in streamline distribution was observed (a quite similar approach was used) (Corrivetti et al., 2019).

Remarkably, a high degree of anatomo-functional variations was identified across patients at both the cortical and subcortical level. More specifically, four patterns were observed: (i) a contribution to both categories of functions; (ii) a unique contribution to semantic cognition; (iii) a unique contribution to mentalizing; (iv) no contribution at all-knowing that mentalizing was over-represented compared with visual semantic cognition. Before elaborating further on this finding, two notes of caution are necessary. First, the fact that no response was identified for either mentalizing or semantics (or both), and most notably at the subcortical level, does not necessary mean that the IFOF was not implicated. Indeed, as patients were operated on according to the individual functional margins (i.e., the surgery procedure stopped when a functional response was repeatedly evoked during stimulation), some IFOF fibers were not stimulated, typically those running in the deeper component of the tract. Second, the pattern "no contribution at all" is quite artificial because uniquely appropriate when considering the cortical and subcortical levels, independently. Indeed, according to the inclusion criteria, patients took place in this study if at least one positive response was observed during the mapping. In other words, a few patients could show a functional response at the cortical level but not at the subcortical level and vice versa; this is not surprising because again all IFOF fibers were not stimulated and only the exposed cortical surface during surgery was stimulated. This implies, in the latter case, that some important cortical sites could have been missed because not accessible to the surgery procedure. With these limits in mind, our results nevertheless suggest an across-subject anatomo-functional variation that may be related to hemispheric dominance. This is especially reminiscent of a study by Catani et al. (2007) in which interhemispheric differences were found in the WM connections of the language network, from strongly left lateralized to bilateral symmetrical distribution. Consequently, a valuable extension of our study would be to assess to what extent semantic and mentalizing performances are related to leftright differences in network topology by combining fMRI and diffusion imaging in healthy participants. Besides, it is worth recalling that handedness may influence the interindividual variations we discussed above, as subcortical sites for mentalizing were less frequent in left-handed vs. right-handed patients.

Last, a few cortical and subcortical sites were associated with both types of functional responses. This result can be interpreted in two ways: either both functional networks were impaired by stimulation, meaning that the spatial resolution of stimulation was not enough to mark topological differences, or a more domain-general control or attention network was temporary disrupted. The latter explanation has to be prioritized, as the aspecific sites were observed uniquely at the cortical level in dlPFC, a region which is known to be involved in such networks (Chein and Schneider, 2005; Duncan, 2010). Besides, at the subcortical level where amodal sites were also observed, the IFOF is believed to support some kinds of amodal processing, especially in the context of semantic cognition (Moritz-Gasser et al., 2013).

The current study had some limitations. First, the positioning of anatomical sites relied on the use of anatomical landmarks identifiable on structural MRI and intraoperative photos. This procedure could lead to potential inaccuracies, but we showed an excellent inter-rater reliability in this mapping work - as in another recent work (Herbet et al., 2019). Second, a templatebased approach was adopted to generate streamline tractograms from stimulation sites. Admittedly, the use of patient diffusion data would have been better. However, there are important difficulties to perform streamline tractography in subcortical structures impaired by lesional infiltrations. Third, low-grade gliomas are disproportionally located in paralimbic areas, implying that posterior structures were less stimulated. A larger set of posterior sites would have certainly help better dissociating the IFOF sublayers. Last, even if the IFOF is considered as a poorly compensable tract (Herbet et al., 2016), we cannot rule out that the possibility that neuroplastic compensations occurred.

In brief, the current study not only confirms that the network mediated by the IFOF is implied in both visual semantic cognition and mentalizing, but also suggests that the tract is dissociable into two functional strata according to a ventral (semantic) and dorsal (mentalizing) compartmentalization. Although this general finding needs to be replicated in healthy participants with other imaging modalities (e.g., coupling navigated transcranial magnetic stimulation with diffusion MRI to track white matter pathways from cortical sites), from a clinical standpoint, they are of central interest for the surgical planning of patients presenting with a tumor in the vicinity of this anatomical connectivity.

\section{DATA AVAILABILITY STATEMENT}

The original contributions presented in the study are included in the article/supplementary material, further inquiries can be directed to the corresponding author/s.

\section{ETHICS STATEMENT}

Ethical review and approval was not required for the study on human participants in accordance with the local legislation and 
institutional requirements. The patients/participants provided their written informed consent to participate in this study.

\section{AUTHOR CONTRIBUTIONS}

$\mathrm{AR}, \mathrm{HD}$, and $\mathrm{GH}$ : conception and design of the study. AR, A-LL, HD, and GH: acquisition and analysis of data and drafting a significant portion of the manuscript and figures. JD and $\mathrm{SN}$ : analysis of data. $\mathrm{SN}, \mathrm{HD}$, and $\mathrm{GH}$ : critical review of the

\section{REFERENCES}

Almairac, F., Herbet, G., Moritz-Gasser, S., de Champfleur, N. M., and Duffau, H. (2015). The left inferior fronto-occipital fasciculus subserves language semantics: a multilevel lesion study. Brain Struct. Funct. 220, 1983-1995. doi: 10.1007/s00429-014-0773-1

Baron-Cohen, S., Wheelwright, S., Hill, J., Raste, Y., and Plumb, I. (2001). The "Reading the Mind in the Eyes" test revised version: a study with normal adults, and adults with Asperger syndrome or high-functioning autism. J. Child Psychol. Psychiatry 42, 241-251. doi: 10.1111/1469-7610.00715

Burdach, K. F. (1822). Vom Baue Und Leben Des Gehirns, Dyk. 2.

Catani, M., Allin, M. P. G., Husain, M., Pugliese, L., Mesulam, M. M., Murray, R. M., et al. (2007). Symmetries in human brain language pathways correlate with verbal recall. Proc. Natl. Acad. Sci. U.S.A. 104, 17163-17168. doi: 10.1073/ pnas.0702116104

Catani, M., Howard, R. J., Pajevic, S., and Jones, D. K. (2002). Virtual in vivo interactive dissection of white matter fasciculi in the human brain. NeuroImage 17, 77-94. doi: 10.1006/nimg.2002.1136

Caverzasi, E., Papinutto, N., Amirbekian, B., Berger, M. S., and Henry, R. G. (2014). Q-ball of inferior fronto-occipital fasciculus and beyond. PloS One 9:e100274. doi: 10.1371 /journal.pone.0100274

Chein, J. M., and Schneider, W. (2005). Neuroimaging studies of practice-related change: fMRI and meta-analytic evidence of a domain-general control network for learning. Brain Res. Cogn. Brain Res. 25, 607-623. doi: 10.1016/j.cogbrainres. 2005.08.013

Corrivetti, F., Thiebaut de Schotten, M., Poisson, I., Froelich, S., Descoteaux, M., Rheault, F., et al. (2019). Dissociating motor-speech from lexico-semantic systems in the left frontal lobe: insights from a series of 17 awake intraoperative mappings in glioma patients. Brain Stuct. Funct. 224, 1151-1165. doi: 10.1007/ s00429-019-01827-7

Crespi, C., Cerami, C., Dodich, A., Canessa, N., Arpone, M., Iannaccone, S., et al. (2014). Microstructural white matter correlates of emotion recognition impairment in Amyotrophic Lateral Sclerosis. Cortex J. Devoted Study Nerv. Syst. Behav. 53, 1-8. doi: 10.1016/j.cortex.2014.01.002

Curran, E. J. (1909). A new association fiber tract in the cerebrum with remarks on the fiber tract dissection method of studying the brain. J. Comp. Neurol. Psychol. 19, 645-656. doi: 10.1002/cne.920190603

Dejerine, J., and Dejerine-Klumpke, A. (1895). Anatomie des Centres Nerveux. Paris: Rueff.

Duda, R. O., and Hart, P. E. (1973). Pattern Classification and Scene Analysis. New York, NY: John Wiley \& Sons Inc.

Duffau, H. (2015). Stimulation mapping of white matter tracts to study brain functional connectivity. Nat. Rev. Neurol. 11, 255-265. doi: 10.1038/nrneurol. 2015.51

Duffau, H., Gatignol, P., Mandonnet, E., Peruzzi, P., Tzourio-Mazoyer, N., and Capelle, L. (2005). New insights into the anatomo-functional connectivity of the semantic system: a study using cortico-subcortical electrostimulations. Brain J. Neurol. 128, 797-810. doi: 10.1093/brain/awh423

Duncan, J. (2010). The multiple-demand (MD) system of the primate brain: mental programs for intelligent behaviour. Trends Cogn. Sci. 14, 172-179. doi: 10.1016/ j.tics.2010.01.004

Grosse Wiesmann, C., Schreiber, J., Singer, T., Steinbeis, N., and Friederici, A. D. (2017). White matter maturation is associated with the emergence of Theory of Mind in early childhood. Nat. Commun. 8:14692. doi: 10.1038/ncomms14692 manuscript. All authors contributed to the article and approved the submitted version.

\section{ACKNOWLEDGMENTS}

AR would like to thank Johan Pallud for his valuable advice and the Nuovo-Soldati Foundation for Cancer Research for their support.

Hau, J., Sarubbo, S., Perchey, G., Crivello, F., Zago, L., Mellet, E., et al. (2016). Cortical terminations of the inferior Fronto-Occipital and uncinate fasciculi: anatomical stem-based virtual dissection. Front. Neuroanat. 10:58. doi: 10.3389/ fnana.2016.00058

Hennig, C. (2020). fpc: Flexible Procedures for Clustering. Available online at: https: //CRAN.R-project.org/package=fpc (accessed December 6, 2020).

Herbet, G., and Duffau, H. (2020). Revisiting the functional anatomy of the human brain: toward a meta-networking theory of cerebral functions. Physiol. Rev. 100, 1181-1228. doi: 10.1152/physrev.00033.2019

Herbet, G., Lafargue, G., Bonnetblanc, F., Moritz-Gasser, S., Menjot de Champfleur, N., and Duffau, H. (2014). Inferring a dual-stream model of mentalizing from associative white matter fibres disconnection. Brain J. Neurol. 137, 944-959. doi: 10.1093/brain/awt370

Herbet, G., Lafargue, G., Moritz-Gasser, S., Bonnetblanc, F., and Duffau, H. (2015). Interfering with the neural activity of mirror-related frontal areas impairs mentalistic inferences. Brain Struct. Funct. 220, 2159-2169. doi: 10. 1007/s00429-014-0777-x

Herbet, G., Maheu, M., Costi, E., Lafargue, G., and Duffau, H. (2016). Mapping neuroplastic potential in brain-damaged patients. Brain J. Neurol. 139, 829-844. doi: 10.1093/brain/awv394

Herbet, G., Moritz-Gasser, S., and Duffau, H. (2017a). Direct evidence for the contributive role of the right inferior fronto-occipital fasciculus in non-verbal semantic cognition. Brain Struct. Funct. 222, 1597-1610. doi: 10.1007/s00429016-1294-x

Herbet, G., Moritz-Gasser, S., Lemaitre, A.-L., Almairac, F., and Duffau, H. (2019). Functional compensation of the left inferior longitudinal fasciculus for picture naming. Cogn. Neuropsychol. 36, 140-157. doi: 10.1080/02643294.2018. 1477749

Herbet, G., Yordanova, Y. N., and Duffau, H. (2017b). Left spatial neglect evoked by electrostimulation of the right inferior fronto-occipital fasciculus. Brain Topogr. 30, 747-756. doi: 10.1007/s10548-017-0574-y

Herbet, G., Zemmoura, I., and Duffau, H. (2018). Functional anatomy of the inferior longitudinal fasciculus: from historical reports to current hypotheses. Front. Neuroanat. 12:77. doi: 10.3389/fnana.2018.00077

Howard, D., Patterson, K. E., and Company, T. V. T. (1992). The Pyramids and Palm Trees Test: a Test of Semantic Access From Words and Pictures. Bury St Edmunds: Thames Valley Test Company.

Latini, F., Mårtensson, J., Larsson, E.-M., Fredrikson, M., Åhs, F., Hjortberg, M., et al. (2017). Segmentation of the inferior longitudinal fasciculus in the human brain: a white matter dissection and diffusion tensor tractography study. Brain Res. 1675, 102-115. doi: 10.1016/j.brainres.2017.09.005

Marino Dávolos, J., Arias, J. C., and Jefferies, E. (2020). Linking individual differences in semantic cognition to white matter microstructure. Neuropsychologia 141:107438. doi: 10.1016/j.neuropsychologia.2020.10 7438

Martino, J., Brogna, C., Robles, S. G., Vergani, F., and Duffau, H. (2010). Anatomic dissection of the inferior fronto-occipital fasciculus revisited in the lights of brain stimulation data. Cortex J. Devoted Study Nerv. Syst. Behav. 46, 691-699. doi: 10.1016/j.cortex.2009.07.015

Mirman, D., Chen, Q., Zhang, Y., Wang, Z., Faseyitan, O. K., Coslett, H. B., et al. (2015). Neural organization of spoken language revealed by lesion-symptom mapping. Nat. Commun. 6:6762. doi: 10.1038/ncomms7762

Mori, S., Frederiksen, K., van Zijl, P. C. M., Stieltjes, B., Kraut, M. A., Solaiyappan, M., et al. (2002). Brain white matter anatomy of tumor patients evaluated 
with diffusion tensor imaging. Ann. Neurol. 51, 377-380. doi: 10.1002/ana. 10137

Mori, S., Wakana, S., van Zijl, P. C. M., and Nagae-Poetscher, L. M. (2005). Mri Atlas Of Human White Matter, 1st Edn. Amsterdam: Elsevier Science Ltd.

Moritz-Gasser, S., Herbet, G., and Duffau, H. (2013). Mapping the connectivity underlying multimodal (verbal and non-verbal) semantic processing: a brain electrostimulation study. Neuropsychologia 51, 1814-1822. doi: 10.1016/j. neuropsychologia.2013.06.007

Ojemann, G., and Mateer, C. (1979). Human language cortex: localization of memory, syntax, and sequential motor-phoneme identification systems. Science 205, 1401-1403. doi: 10.1126/science.472757

Philippi, C. L., Mehta, S., Grabowski, T., Adolphs, R., and Rudrauf, D. (2009). Damage to association fiber tracts impairs recognition of the facial expression of emotion. J. Neurosci. 29, 15089-15099. doi: 10.1523/JNEUROSCI.0796-09. 2009

Rech, F., Herbet, G., Gaudeau, Y., Mézières, S., Moureau, J.-M., Moritz-Gasser, S., et al. (2019). A probabilistic map of negative motor areas of the upper limb and face: a brain stimulation study. Brain J. Neurol. 142, 952-965. doi: 10.1093/brain/awz021

Sarubbo, S., De Benedictis, A., Maldonado, I. L., Basso, G., and Duffau, H. (2013). Frontal terminations for the inferior fronto-occipital fascicle: anatomical dissection, DTI study and functional considerations on a multicomponent bundle. Brain Struct. Funct. 218, 21-37. doi: 10.1007/s00429-0110372-3

Sarubbo, S., Petit, L., De Benedictis, A., Chioffi, F., Ptito, M., and Dyrby, T. B. (2019). Uncovering the inferior fronto-occipital fascicle and its topological organization in non-human primates: the missing connection for language evolution. Brain Struct. Funct. 224, 1553-1567. doi: 10.1007/s00429-01901856- 2

Sarubbo, S., Tate, M., De Benedictis, A., Meler, S., Moritz-Gasser, S., Herbet, G., et al. (2020). Mapping critical cortical hubs and white matter pathways by direct electrostimulation: an original functional atlas of the human brain. Neuroimage 205:166237. doi: 10.1016/j.neuroimage.2019.116237

Schmahmann, J. D., and Pandya, D. N. (2007). The complex history of the fronto-occipital fasciculus. J. Hist. Neurosci. 16, 362-377. doi: 10.1080/ 09647040600620468

Tate, M. C., Herbet, G., Moritz-Gasser, S., Tate, J. E., and Duffau, H. (2014). Probabilistic map of critical functional regions of the human cerebral cortex: Broca's area revisited. Brain J. Neurol. 137, 2773-2782. doi: 10.1093/brain/ awu168

Thiebaut de Schotten, M., Dell'Acqua, F., Valabregue, R., and Catani, M. (2012). Monkey to human comparative anatomy of the frontal lobe association tracts. Cortex J. Devoted Study Nerv. Syst. Behav. 48, 82-96. doi: 10.1016/j.cortex.2011. 10.001
Thomas, C., Avidan, G., Humphreys, K., Jung, K., Gao, F., and Behrmann, M. (2009). Reduced structural connectivity in ventral visual cortex in congenital prosopagnosia. Nat. Neurosci. 12, 29-31. doi: 10.1038/nn.2224

Trolard, P. (1906). Le faisceau longitudinal inférieur du cerveau. Rev. Neurol. (Paris) 14, 440-446.

Urbanski, M., Thiebaut de Schotten, M., Rodrigo, S., Catani, M., Oppenheim, C., Touzé, E., et al. (2008). Brain networks of spatial awareness: evidence from diffusion tensor imaging tractography. J. Neurol. Neurosurg. Psychiatry 79, 598-601. doi: 10.1136/jnnp.2007.126276

Vandermosten, M., Boets, B., Poelmans, H., Sunaert, S., Wouters, J., and Ghesquière, P. (2012). A tractography study in dyslexia: neuroanatomic correlates of orthographic, phonological and speech processing. Brain J. Neurol. 135, 935-948. doi: 10.1093/brain/awr363

Wang, K., Li, X., Huang, R., Ding, J., Song, L., and Han, Z. (2020). The left inferior longitudinal fasciculus supports orthographic processing: evidence from a lesion-behavior mapping analysis. Brain Lang. 201, 104721. doi: 10.1016/ j.bandl.2019.104721

Wu, Y., Sun, D., Wang, Y., and Wang, Y. (2016). Subcomponents and connectivity of the inferior fronto-occipital fasciculus revealed by diffusion spectrum imaging fiber tracking. Front. Neuroanat. 10:88. doi: 10.3389/fnana.2016.00088

Yeh, F.-C., Liu, L., Hitchens, T. K., and Wu, Y. L. (2017). Mapping immune cell infiltration using restricted diffusion MRI. Magn. Reson. Med. 77, 603-612. doi: 10.1002/mrm.26143

Yeh, F.-C., and Tseng, W.-Y. I. (2011). NTU-90: a high angular resolution brain atlas constructed by $\mathrm{q}$-space diffeomorphic reconstruction. NeuroImage 58, 91-99. doi: 10.1016/j.neuroimage.2011.06.021

Yeh, F.-C., Wedeen, V. J., and Tseng, W.-Y. I. (2010). Generalized q-sampling imaging. IEEE Trans. Med. Imaging 29, 1626-1635. doi: 10.1109/TMI.2010. 2045126

Yordanova, Y. N., Duffau, H., and Herbet, G. (2017). Neural pathways subserving face-based mentalizing. Brain Struct. Funct. 222, 3087-3105. doi: 10.1007/ s00429-017-1388-0

Conflict of Interest: The authors declare that the research was conducted in the absence of any commercial or financial relationships that could be construed as a potential conflict of interest.

Copyright (c) 2021 Roux, Lemaitre, Deverdun, Ng, Duffau and Herbet. This is an open-access article distributed under the terms of the Creative Commons Attribution License (CC BY). The use, distribution or reproduction in other forums is permitted, provided the original author(s) and the copyright owner(s) are credited and that the original publication in this journal is cited, in accordance with accepted academic practice. No use, distribution or reproduction is permitted which does not comply with these terms. 